Ebisu

Études japonaises

Études japonaises

$54 \mid 2017$

L'après-guerre des intellectuels japonais

\title{
Sur les origines de la nouvelle gauche et des mouvements de contre-culture. Nakai Masakazu et la « pensée contemporaine »
}

新左翼とカウンターカルチャーの起源について一中井正一と「現代 思想」

On the Origins of the New Left and Counterculture Movements. Nakai Masakazu and "Contemporary Thought"

\section{Michael Lucken}

\section{OpenEdition}

Journals

Édition électronique

URL : http://journals.openedition.org/ebisu/2002

DOI : $10.4000 /$ ebisu.2002

ISSN : 2189-1893

Éditeur :

Institut français de recherche sur le Japon (UMIFRE 19 MAEE-CNRS), Maison franco-japonaise

Édition imprimée

Date de publication : 19 décembre 2017

Pagination : 47-78

ISSN : $1340-3656$

\section{Référence électronique}

Michael Lucken, «Sur les origines de la nouvelle gauche et des mouvements de contre-culture. Nakai Masakazu et la « pensée contemporaine » ». Ebisu [En ligne], 54 | 2017, mis en ligne le 19 décembre 2017, consulté le 20 avril 2019. URL : http://journals.openedition.org/ebisu/2002 ; DOI : 10.4000/ ebisu.2002 


\section{Sur les origines de la nouvelle gauche et des mouvements de contre-culture}

Nakai Masakazu et la «pensée contemporaine »

\section{Michael LuCKen}

新左翼とカウンターカルチャーの起源について一

中井正一と「現代思想」

ミカエル・リュケン

On the Origins of the New Left and Counterculture Movements. Nakai Masakazu and "Contemporary Thought" Michael Lucken

\Mots-clés : Nakai Masakazu, Tsurumi Shunsuke, École de Francfort, Nouvelle Gauche, théorie critique, Marx, Lénine, contre-culture, $k i$.

L’auteur : Michael Lucken (né en 1969) est professeur à l'Institut national des langues et civilisations orientales (Inalco, Paris). Historien spécialiste de l'art et de la culture du Japon moderne, il a publié, entre autres, Nakai Masakazu. Naissance de la théorie critique au Japon (Presses du réel, 2015). Il dirige depuis 2014 le Centre d'Études Japonaises de l'Inalco.

Résumé : La pensée de Nakai Masakazu
(1900-1952) exerce depuis plus d'un demi-siècle une influence discrète, mais persistante, sur la culture japonaise. La carrière de Nakai commença avec l'étude de Kant et, à travers Kant, de Cassirer. Mais il y a également une dimension marxiste dans les textes de Nakai. Dans cet article, qui porte principalement sur ses liens avec les penseurs de l'École de Francfort qui furent présentés dans la revue Sekai bunka (Culture du monde) dès 1935, j'essaierai de comprendre comment, et dans quelle mesure, la pensée de Nakai peut être considérée, comme elle l'est depuis la fin des années 1950, comme un modèle de contemporanéité. 
キーワード

中井正一、鶴見俊輔、フランクフルト学派、 新左翼、批判理論、マルクス、レーニン、 カウンターカルチャー、気

\section{著者}

ミカエル・リュケン (1969 年生まれ) はフランス 東洋言語文化大学 (イナルコ) 教授、同大学日 本研究所所長。専門は日本近代美術史、思想史。

\section{要旨}

哲学者中井正一 $(1900$ - 1952) の思想は、
半世紀以上にわたり、日本の文化にひそかに影 響を与え続けている。中井はカントを学ぶこと からはじめ、そしてカントを通じてカッシーラー を発見した。しかし一方で中井の著作にはまた、 マルクス主義的な側面もある。本論文では、 1935 年から雑誌『世界文化』で紹介されたフラ ンクフルト学派の思想家たちとの共通点に注目し つつ、1950 年代から今日にいたるまで、いかに して中井の思想が現代性のモデルとして捉えられ るようになったのかを考察する。 \eywords: Nakai Masakazu, Tsurumi Shunsuke, Frankfurt School, New Left, critical theory, Marx, Lenin, counter-culture, $k i$.

The Author: Michael Lucken (1969-) is a professor at the National Institute of Oriental Languages and Civilizations (Inalco) in Paris. He is the author of Imitation and Creativity in Japanese Arts: from Kishida Ryūsei to Miyazaki Hayao (Columbia University Press, 2016), The Japanese and the War 1937-1945 (Columbia University Press, 2017), among others.
Abstract: The thought of philosopher Nakai Masakazu (1900-1952) has exerted a quiet but enduring influence on Japanese culture for more than half a century. Nakai began his career studying Kant, discovering in the process Cassirer. But there is also a Marxist dimension to Nakai's writings. This paper focuses on his links to thinkers from the Frankfurt School, who were presented in Sekai bunka (World Culture) as early as 1935. It attempts to understand how and in what sense Nakai's thought can be said - as has been the case since the late 1950 s - to provide a model of contemporaneity. 


\title{
Sur les origines de la nouvelle gauche et des mouvements de contre-culture
}

\author{
Nakai Masakazu et la «pensée contemporaine »
}

Michael LUCKEN*

Nakai Masakazu 中井正一 (ou Nakai Shōichi, 1900-1952) est un philosophe dont la pensée travaille secrètement la culture japonaise depuis plus d'un demi-siècle. Associé à l'aile gauche de l'école de Kyoto, il n'a jamais été célèbre, mais il n'a jamais non plus été oublié. Quelle que soit l'époque, il se rencontre toujours des intellectuels et des artistes qui s'appuient sur son œuvre pour ouvrir de nouvelles pistes. C'est comme s'il était étranger au phénomène de la mode, comme s'il était toujours d'aujourd'hui sans jamais l'être vraiment.

La critique du Japon moderne (Kindai Nihon no hihyō 近代日本の批評) est un livre d'entretiens publié en 1997 dont l'objectif était d'interroger l'héritage des intellectuels du $\mathrm{xx}^{\mathrm{e}}$ siècle. Dans un passage consacré aux années de guerre (1930-1945), le sémiologue et critique de cinéma Hasumi Shigehiko 蓮實重彦 (né en 1936) revient à plusieurs reprises sur le rôle historique de Nakai, l'originalité de sa réflexion sur l'art et les médias, les limites de son concept de subjectivité collective. Il y souligne notamment la proximité entre ce que dit Nakai de la société de consommation de masse, où les gens n'achètent en guise d'objets que des images dont ils ne comprennent pas le sens, et ce que soutiendra Baudrillard près d'un demi-siècle plus tard (Karatani 1997 : 147). Hasumi en conclut plus loin : "On peut même dire que Nakai anticipe tout ce qu'on appelle couramment la "pensée

* INALCO. 
contemporaine” "(Karatani $1997: 256)^{1}$. Cette remarque constitue mon point de départ : à quoi renvoie ici l'expression "pensée contemporaine " ? Quelles en sont les bornes, les valeurs, les dynamiques? En quoi l'œuvre de Nakai fournit-elle un modèle de contemporanéité ?

Ce qui est contemporain à un moment donné ne l'est plus quelques générations plus tard. C'est comme une règle pochoir qui glisse vers le présent au rythme du temps biologique. Toutefois, ce glissement n'est pas linéaire, il se fait généralement par à-coups. Au quotidien, la chaîne de mouillage se dévide automatiquement. Jusqu'au jour où un vent violent fait lâcher l'ancre qui dérape brusquement. Depuis plusieurs décennies, 1945 sert de principal point d'ancrage. Mais en dépit de tous les efforts pour maintenir vivant le souvenir de cette date, la tension, inexorablement, se fait de plus en plus forte sur la chaîne. Alors on prospecte et l'on essaie de jeter d'autres ancres pour soulager la première. Tel est l'horizon de cet essai : contribuer à redéfinir l'origine de la contemporanéité tout en conservant une place fondatrice à la fin de la Seconde Guerre mondiale, autrement dit chercher une manière de refonder le présent autrement que par l'arrachement et la violence.

Cet article, écrit dans un mouvement essentiellement rétrospectif, part de la réception de l'œuvre de Nakai dans les années 1960, période qui a bouleversé les mœurs, l'esthétique et les rapports sociaux. Cette première partie se poursuit par une exploration des liens entre Nakai et les philosophes qui ont marqué l'époque, à commencer par Marx et les penseurs de l'école de Francfort. Ramenés par ce biais jusque dans les années 1930, nous terminerons par une analyse du concept de $k i$ 気/機 chez Nakai comme moment d'embrayage entre théorie et praxis, entre conscience historique et action individuelle.

\section{Nakai et les années 1960}

Hasumi Shigehiko, formé en littérature française à l'université de Tokyo, a été l'un des premiers traducteurs de Deleuze, Derrida et Barthes en japonais. Il fut aussi un important théoricien du cinéma et contribua à former

1. 中井は俗にいう「現代思想」を全部先取りしているとさえ言える。 
toute une génération de réalisateurs, à commencer par Kurosawa Kiyoshi 黒沢清 (né en 1955) et Aoyama Shinji 青山真治 (né en 1964). Comme il le rapporte dans un entretien, il eut la chance de rencontrer Nakai juste avant la mort de ce dernier à l'occasion d'une réception où ses parents étaient invités. Le philosophe, qui était alors vice-président de la bibliothèque de la Diète ${ }^{2}$, lui donna à cette occasion le livre qu'il venait de publier, Introduction à l'esthétique (Bigaku nyūmon 美学入門), dont le cœur est une réflexion sur le cinéma (Nakai 1951: 68-87) ${ }^{3}$.

Il existe plusieurs anecdotes de ce type. Nakai a profondément marqué de jeunes intellectuels qu'il a connus soit à Hiroshima entre 1945 et 1948, soit à Tokyo entre 1948 et sa mort en 1952. La réception de son œuvre dans les années 1960 s'est faite en premier lieu à travers des critiques, nés pour la plupart entre 1920 et 1935, qui ont été touchés par sa pensée et son action, mais aussi par son charisme (Yoshida 2003 : 8-10). Parmi ceuxci, on citera Tsurumi Shunsuke 鶴見俊輔 (1922-2015), Hariu Ichirō 針生 一郎 (1925-2010), Suzuki Tadashi 鈴木正 (né en 1928) et Kurita Isamu 栗田勇 (né en 1929). Mais il faut ajouter aussi les noms de ceux qui l'ont connu avant-guerre à Kyoto, et qui étaient encore actifs dans les années 1960, à commencer par le philosophe Kuno Osamu 久野収 (1910-1999), le linguiste francisant Shinmura Takeshi 新村猛 (1905-1992), le romancier Noma Hiroshi 野間宏 (1915-1991) et le critique de cinéma Imamura Taihei 今村太平 (1911-1986).

Il y a deux facettes à l'intérêt manifesté pour l'œuvre de Nakai dans les années 1960. La première est d'ordre politique, la seconde est plus spécifiquement esthétique, même si la dimension politique est là encore prégnante. C'est en 1959 qu'on peut faire débuter les études sur Nakai avec en particulier un texte de Tsurumi Shunsuke intitulé « La matrice de fermentation de la pensée » (Shisō no hakkō botai 思想の発酵母胎; Tsurumi 1959:

2. Nakai fut nommé vice-président de la bibliothèque de la Diète en avril 1948, sur recommandation de Hani Gorō 羽仁五郎 (1901-1983). Il joua alors un rôle déterminant dans la rédaction de la Loi sur les bibliothèques (Satō 1992 : 7-20). Cette loi, adoptée en 1950, donna des usages communs à toutes les institutions concernées et permit de hiérarchiser les missions des bibliothèques, en subordonnant clairement la conservation des documents à l'information du public.

3. Anecdote rapportée par Hasumi lors d'un entretien public au Aoyama Book Center (Tokyo) le 8 septembre 2007. 
30-39). 1959 fut au Japon une année charnière sur le plan politique et économique. En réaction au projet du gouvernement de reconduire le traité de sécurité nippo-américain, se cristallisa à partir de mars un puissant mouvement contestataire piloté par la Ligue des communistes (Kyōsanshugisha dōmei 共産主義者同盟), un groupe dissident du PCJ, d'obédience trotskiste, fondé en décembre 1958. C'est le début d'une période d'une douzaine d'années caractérisées par des manifestations violentes qui culmina en 1968-1969 avec les révoltes étudiantes et les actions contre la guerre du Vietnam. Mais elle correspond aussi au début de la période de très haute croissance qui court jusqu'en 1970. C'est notamment l'année où le PNB japonais a le plus progressé $(+17,5 \%)$. Cette vitalité économique se traduisit par de grands projets énergétiques (centrales nucléaires, barrages hydroélectriques), le lancement de vastes chantiers d'infrastructure ferroviaires et routiers ainsi que l'édification de grands ensembles d'immeubles standardisés pour loger les classes moyennes. Elle s'accompagna en outre de la diffusion rapide de la télévision qui non seulement bouleversa le rapport à l'information, mais eut des effets profonds sur la perception de l'espace. Le regain d'intérêt pour Nakai est profondément lié à ce contexte paradoxal, entre prospérité et révolte sociale.

Dans son article de 1959, Tsurumi Shunsuke développe sa réflexion à partir d'un texte de Nakai intitulé "La logique des comités " (Iinkai no ronri 委員会の論理, 1936) ${ }^{4}$ qu'il présente comme «sans doute l'une des grandes réalisations de l'histoire de la philosophie japonaise » (Tsurumi 1959: 32) . Dans ce texte, explique-t-il, Nakai a montré que l'idée d'une logique abstraite transcendant les phénomènes n'est rien d'autre qu'une construction humaine, autrement dit que la logique elle-même possède une histoire. La civilisation humaine a d'abord connu la logique parlée, avec notamment

4. "La logique des comités " parut pour la première fois dans la revue Sekai bunka 世界文化 (Culture du monde), 13, janvier 1936 : 2-17；14, février 1936 : 16-33；15, mars $1936: 12-25$. Une traduction annotée en français par l'auteur est parue dans European Journal of Japanese Philosophy, 1, 2016 : 289-357.

5. 日本哲学史上の一つの重大な達成であろう。On notera que «La logique des comités » est présenté en termes très proches dans Nihon no meicho. Kindai no shisō 日本の名著: 近代の思想 (Les grands textes du Japon. La pensée moderne), un ouvrage de référence édité par le spécialiste de littérature française Kuwabara Takeo 桑原武夫 (1904-1988) (Kuwabara 1962: 195). 
les Grecs, avant que celle-ci ne se transforme pour devenir écrite avec les Romains, puis technique à la Renaissance, et enfin industrielle au XIX ${ }^{\mathrm{e}}$ siècle. À chaque phase correspond un média qui la caractérise : la joute oratoire pour la première, le manuscrit pour la seconde, l'imprimerie pour la troisième, le comité pour la dernière (Tsurumi 1959 : 33). Les commissions gouvernementales, les instituts de recherche industriels, les organisations financières et internationales sont autant de manifestations différentes de ce que Nakai appelle un comité.

Mais, observe Tsurumi, la défaite de 1945 a balayé les structures établies et le Japon s'est reconstruit par petits cercles, dans un geste de survie. Or ces petits cercles qu'on retrouve dans tous les domaines - et qui correspondent au niveau artistique à des groupes comme Jikken kōbō 実験工房 et Gutai 具体 - sont fondés principalement sur le dialogue et renvoient à un stade ancien de la culture. Ils sont donc incapables de faire face aux organisations complexes (réseaux politiques, consortiums économiques, mass-media) que les puissants ont reconstituées depuis la fin des hostilités. Dans un monde où, comme le dit Nakai, l'homme a perdu l'accès à la compréhension de la généralité des concepts des produits qu'il consomme du fait des mensonges inhérents à la logique marchande, les groupes disparates ne sont pas de taille à lutter, et les individus isolés encore moins (Nakai 1981a : 96-99).

Toutefois Tsurumi ne se contente pas de réclamer une restructuration des groupes existants au sein d'ensembles plus larges. Toujours en s'appuyant sur Nakai, il souligne que le principe de délégation (futakusei 付託性) sur lequel reposent les grandes organisations contemporaines constitue le point de fragilité du système qui se corrompt nécessairement si un contre-pouvoir critique n'est pas établi. Ce qui est à l'origine une mission confiée à des représentants se naturalise et devient un privilège. Il ne s'agit donc pas pour la gauche de choisir entre l'affiliation à un parti ou la constitution de structures plus horizontales comme des fédérations, il faut au contraire que les deux formes d'organisations, qui correspondent historiquement à la classe ouvrière et à la classe moyenne, coexistent et s'aiguillonnent mutuellement pour le bénéfice de tous (Tsurumi 1959 : 34-35).

L'intérêt de Tsurumi pour la pensée de Nakai s'accompagne d'une réappréciation de son rôle historique dans les années 1930, période au cours de laquelle ce dernier s'est impliqué activement dans le mouvement antifasciste (Tsurumi 1963 : 84-89). Au début des années 1960, Nakai émerge donc non seulement comme un théoricien, mais aussi comme un homme 
qui a mis en pratique ses idées en menant la lutte contre l'éviction du professeur Takigawa Yukitoki 㴰川幸辰 (1891-1962) de l'université de Kyoto en 1933, et surtout en publiant Doyōbi 土曜日 (Samedi) et Sekai bunka 世界文化 (Culture du monde), deux revues qui, jusqu’à son incarcération fin $1937^{6}$, ont défendu, par le biais de l'information culturelle, la création d'un front populaire pacifiste et internationaliste au Japon (Baba 2009 : 121-195; Lucken 2015 : 153-162) ${ }^{7}$. Il a d'autant plus suscité l'intérêt qu'il n'a jamais été communiste, ce qui faisait de lui un possible modèle pour tous les étudiants et intellectuels qui cherchaient alors à développer une " nouvelle gauche" (shin-sayoku 新左翼).

Le deuxième aspect de la réception de Nakai dans les années 1960 concerne le volet plus spécifiquement esthétique de son œuvre. Ce sont dans les domaines du cinéma et de l'architecture que ses travaux ont été les plus remarqués, ce qui n'est pas surprenant puisque la question du cinéma est très présente chez lui, et qu'il s'est par ailleurs beaucoup interrogé sur le rapport de l'homme à l'espace. Le critique Kawazoe Noboru 川添登 (1926-2015), l'un des fondateurs du mouvement Métabolismeメタボリズム qui a bouleversé l'histoire de l'architecture japonaise, écrit par exemple : "Lorsque je voulais réfléchir un tant soit peu sérieusement à ce qu'était l'architecture, je commençais toujours par essayer de relire les textes de Nakai Masakazu» (Kawazoe 1981: 12) ${ }^{8}$.

Kawazoe semble surtout avoir retenu l'interprétation que le philosophe donne de la forme comme moment de retour au réel d'un ensemble de fonctions données. Comme il l'explique dans un livre de 1965, un marteau

6. Nakai fut incarcéré en novembre 1937. Hospitalisé au cours de l'hiver 1939, il attendit ensuite son procès en résidence surveillée. Reconnu coupable par la justice d'avoir enfreint la Loi sur l'ordre public et la sécurité, il fut condamné en 1940 à deux ans de prison et deux ans de travaux forcés avec sursis. Sa peine ayant été purgée en préventive, il ne retourna pas en prison.

7. Tsurumi a revendiqué à plusieurs reprises la filiation entre sa revue Shisō no kagaku 思想の科学 (La science des idées) et ces deux revues créées par Nakai. Cette filiation est incarnée par la présence parmi les sept fondateurs de Shisō no kagaku de Taketani Mitsuo 武谷三男 (1911-2000), physicien, philosophe des sciences et ami de Nakai, qui fut l'un des collaborateurs les plus actifs de Sekai bunka entre 1935 et 1937 (Tsurumi $2009: 5)$.

8. 建築について少しでも深くつつこんで考えようとするときには、必ず中井正一氏の諸論文を読 みかえしてみることから始めた。 
a toujours été composé d'un butoir et d'un manche, mais sa forme a évolué au cours des âges lui permettant d'acquérir de nouvelles fonctions (enlever les clous par exemple). À travers cet exemple, on voit que la forme ne se contente pas de suivre la fonction, elle la précise et l'enrichit dans un constant mouvement d'enrichissement mutuel. Elle ne lui est pas subordonnée, mais coordonnée (Kawazoe 1965 : 225-229). Nakai, qui est cité à plusieurs reprises, a donc aidé Kawazoe à sortir du fonctionnalisme rigide imposé par les modernistes à partir des années 1920.

Dans le domaine du cinéma, plusieurs metteurs en scène associés à la Nouvelle vague, comme Yoshida Kijū 吉田喜重 (né en 1933), Hani Susumu 羽仁進 (né en 1928) et Matsumoto Toshio 松本俊夫 (1932-2017), ont attentivement lu Nakai (Capel 2015 : 84-95). Dans « L'espace et le temps au cinéma» (1972), Yoshida revient longuement sur l'idée nakaïenne que la caméra, par sa capacité à refléter le réel encore mieux que l'œil humain, accentue un double sentiment d'attachement au monde (du fait que les choses se montrent) et de détachement du monde (du fait qu'il faut constamment couper les plans, c'est-à-dire cesser de regarder) (Yoshida 2006 : 264280). Nakai lui a donc servi à mettre en tension la nouvelle objectivité du regard permise par la machine, et une subjectivité qui se découvre et se réalise dans la coupe et le montage. Autrement dit, il lui a fourni des outils conceptuels pour dépasser le réalisme du cinéma documentaire soviétique sans verser pour autant dans le cinéma-fiction et la théâtralité. Les exemples de Kawazoe et de Yoshida suggèrent que l'œuvre de Nakai fut avant tout féconde dans les années 1960 parce qu'elle a permis de réaffirmer l'importance de l'individu, du contingent, du phénomène, non pas contre le collectif, l'histoire ou la fonction, mais avec eux. Cette dialectique fut perçue alors comme l'une des clés possibles de la contemporanéité 9 .

Nakai ne fut cependant pas le seul à être lu dans cette perspective. Le succès de Marcuse à cette époque répond au même besoin de trouver une autre alternative au communisme que le compromis social-démocrate. La

9. On peut mentionner aussi le cas de Matsumoto Toshio qui, en 1964, écrit: "D'après [Nakai], le cinéma est non seulement l'authentique art "contemporain" que le contemporain devait inévitablement engendrer, mais il est, en tant que médium, le mieux à même d'exprimer le contemporain dans son essence " (Matsumoto 2006: 238). Je reprends ici la traduction de Mathieu Capel (Capel 2012 : 229). 
publication en japonais d'Éros et civilisation en 1958, texte qui analyse comment les individus intériorisent les normes répressives, contribua à faire éclore une forme de contestation sociale plus spontanée et plus directement libératrice pour les individus ${ }^{10}$. Plus encore, la traduction des textes du penseur allemand sur les Manuscrits de $1844^{11}$ révélèrent, comme dit Howard, "un Marx "philosophe", "hégélien”, "humaniste", qui semble, de prime abord, avoir peu en commun avec l'auteur du Manifeste communiste et du Capital " (Howard 1970 : 97). Ce nouveau regard sur le marxisme fut non seulement véhiculé par Marcuse, mais aussi par toute l'école de Francfort dont les œuvres commencèrent à être diffusées en japonais dès la fin des années 1950 : Société aliénée et société saine de Fromm fut publié en 1958 ; L'euvre d'art à l'époque de sa reproductibilité technique de Benjamin, en 1965 ; Théorie et pratique de Habermas, en 1969 ; Éclipse de la raison de Horkheimer, en 1970, etc ${ }^{12}$. Les années 1960 sont donc marquées par une pénétration forte de la théorie critique, autrement dit par une relecture dynamique de Marx permettant de rejeter à la fois le stalinisme et la socialdémocratie, mais aussi d'apporter des réponses aux questions soulevées

10. Herbert Marcuse ヘルベルド・マルクーゼ, Erosuteki bunmei エロス的文明 (Eros and Civilization [Éros et Civilisation]), trad. Minami Hiroshi 南博, Tokyo, Kinokuniya shoten 紀伊国屋書店, 1958.

11. Herbert Marcuse, Shoki Marukusu kenkyū : Keizaigaku tetsugaku shukō ni okeru sogairon 初期マルクス研究『経済学・哲学手稿』における疎外論 (Neue Quellen zur Grundlegung des Historischen Materialismus. Interpretation der neuveröffentlichten Manuskripte von Marx [Étude sur le premier Marx : de l'aliénation dans les Manuscrits économicophilosophiques]), trad. Rachi Chikara 良知力 et Ikeda Yūzō 池田優三, Tokyo, Miraisha 未来社, 1961.

12. Erich Fromm エーリッヒ・フロム, Shōki no shakai 正気の社会 (The Sane Society [Société aliénée et société saine]), trad. Katō Masaaki 加藤正明 et Sase Takao 佐瀬隆夫, Tokyo, Shakai shisōsha 社会思想社, 1958 ; Walter Benjamin ヴァルター・ベンヤミン, Fukusei gijutsu jidai no geijutsu 複製技術時代の芸術 (L'oeuvre d'art à l'époque de sa reproductibilité technique), trad. Kawamura Jirō 川村二郎 et al., Tokyo, Kinokuniya shoten, 1965 ; Jürgen Habermas ユルゲン・ハーバーマス, Shakai tetsugaku ronshū: seiji ni okeru riron to jissen 社会哲学論集 政治における理論と実践 (Theorie und Praxis [Théorie et pratique]) vol. 1, trad. Hosoya Sadao 細谷貞雄, Tokyo, Miraisha, 1969 ; Max Horkheimer マックス・ ホルクハイマー, Risei no fushoku 理性の腐蝕 (Eclipse of Reason [Éclipse de la raison]), trad. Yamaguchi Masahiro 山口祐弘, Tokyo, Serika shobō せりか書房, 1970. 
par la consommation de masse et les nouveaux médias ${ }^{13}$. Les ouvrages de réflexion socio-politique de Yoshimoto Takaaki 吉本隆明 (1924-2012) en sont les meilleurs exemples, à commencer par son Karl Marx de 1966 où les textes de jeunesse du penseur allemand occupent une place centrale (Yoshimoto 1966).

Je voudrais montrer à présent que la concomitance de la pénétration des idées de l'école de Francfort et de la redécouverte de Nakai, dont les œuvres complètes commencèrent à être publiées en 1964, n'est pas une coïncidence. Il y a en effet entre ces intellectuels des liens historiques et des références communes qui permettent de mieux comprendre l'origine de la nouvelle gauche et des mouvements de contre-culture au Japon.

\section{Nakai, penseur marxiste?}

Nakai commença ses études à l'université de Kyoto en 1922 où il reçut une solide formation en philosophie. Élève de Fukada Kōsan 深田康算 (1878-1928), il consacra ses premiers travaux de recherche à l'analyse de l'évolution du rapport entre esthétique et technique dans la Critique $d u$ jugement de Kant. Logiquement, il s'intéressa ensuite au fonctionnalisme

13. Dans Japan's New Left Movements, Takemasa Ando écrit: "Considering that Marcuse's works were translated into Japanese in the 1970s and after, it is not reasonable to advocate that Japanese New Leftists read the works carefully in shaping their ideas " (Ando 2013 : 13). Bien qu'Ando ait raison d'affirmer que la nouvelle gauche japonaise ne doit pas être réduite à un épigone des mouvements occidentaux, une demi-douzaine de titres de Marcuse étaient disponibles en japonais dès la fin des années 1960. Outre les ouvrages précités, on peut mentionner les traductions de Reason and Revolution: Hegel and the rise of social theory (Risei to kakumei: Hégeru to shakai riron no köryū 理性と 革命 : ヘーゲルと社会理論の興隆, Iwanami shoten, 1961), Das Ende der Utopie (Yütopia no shūen: kajō, yokuatsu, bōryoku ユートピアの終焉 : 過剩・抑圧・暴力, Gōdō shuppan 合同出版, 1967), Psychoanalyse und Politik (Sei to shi no shōdō 生と死の衝動, Gōdō shuppan, 1969) ou encore Kultur und Gesellschaft (Bunka to shakai 文化と社会, Serika shobō, 2 vol., 1969-1972). L'Homme unidimensionnel (One-Dimensional Man) ne fut traduit en entier qu'en 1974. Cette erreur factuelle, qui fragilise toute l'argumentation d'Ando, non seulement occulte l'immense travail de traduction fait par les intellectuels japonais depuis les années 1930, mais interdit une réflexion nécessaire sur le pourquoi de cet intérêt précoce pour l'école de Francfort. 
de Cassirer que l'on découvrait alors (Lucken 2016 : 81-88). Entre 1928 et 1933 enfin, il fut marqué, comme la plupart des philosophes de sa génération, par la lecture d'Etre et Temps de Heidegger dont il retint l'idée d'une priorité de l'espace intersubjectif de l'être sur l'espace mesurable et quantifiable de la science (Lucken 2014 : 60-65). Kant, Cassirer et, dans une moindre mesure, Heidegger sont dans son œuvre trois références majeures et explicites.

Le rapport de Nakai à Marx est en revanche beaucoup moins clair. Nakai n'a pas participé au mouvement prolétarien au cours des années 1920 et, après-guerre, il n'a pas adhéré au PCJ dont l'existence était pour la première fois légale. Il ne fait par ailleurs que très peu référence de manière explicite à Marx dans ses textes, et il ne s'est jamais affiché comme marxiste. En 1952, une enquête secrète du ministère de la Justice pour le Haut commandement des forces alliées (SCAP) conclut : "Rien n'indique que Nakai Shōichi ait des liens directs ou indirects avec le Parti communiste japonais " (Yoshikawa 1952: 4) ${ }^{14}$. On sait par contre qu'il défendit avec Tosaka Jun 戸坂潤 (1900-1945) l'intérêt du marxisme au cours du débat qui eut lieu en 1929 au sein de l'école de Kyoto (Kōyama 1995 : 85, 167), qu’il participa activement au mouvement coopératif dans les années $1930^{15}$, qu’il fut condamné en 1940 pour des "actes conçus pour remplir les objectifs du Komintern et du parti communiste " (Baba 2009 : 206), qu'il a rejoint le parti socialiste en 1947 et que sa nomination en 1948 à la bibliothèque de

14. Le nom de Nakai apparaît dans les archives américaines dès 1948. Ce document de janvier 1952 est toutefois le seul rapport rédigé que nous ayons trouvé. Il montre que la contestation de la nomination de Nakai comme vice-président, ravivée par les « purges rouges " de 1949-1950, n’a jamais cessé au sein de la bibliothèque de la Diète (Pincus 2006 : 382-392).

15. La répression systématique des mouvements prolétariens à partir de 1928 a entraîné le développement des coopératives de consommation (shōhi kumiai 消費組合) qui furent entre 1930 et 1934 l'un des principaux espaces d'expression des sensibilités de gauche. Entre 1930 et 1932, Nakai dirigea la Coopérative familiale de Kyoto (Kyōto katei shōhi kumiai 京都過程消費組合) qui comprit jusqu'à 650 adhérents parmi lesquels de nombreux artistes et intellectuels, à commencer par Nishida Kitarō 西田幾多郎 (18701945), Tanabe Hajime 田辺元 (1885-1962), Tosaka Jun, Yanagi Muneyoshi 柳宗悦 (1889-1961), Kawai Kanjirō 河井寛次郎 (1890-1966), Tsuda Seifū 津田青楓（18801978) et Suda Kunitarō 須田国太郎 (1891-1961) (Kyōdō kumiai-shi kenkyūkai 2003 ; Satō 2012 : 19-21). 
la Diète fut décriée sur le mode : "Pas question d'un Rouge comme viceprésident !» (Baba 2009 : 307).

Les amis de Nakai et les critiques qui se sont intéressés à son œuvre donnent des interprétations tout aussi contradictoires. Tsurumi écrit en 1959 qu'il est "à peu près clair ${ }^{16}$ " que "La logique des comités " a été conçue sous l'influence d'Un pas en avant, deux pas en arrière de Lénine (Tsurumi 1959 : 33). Plus que tout autre, il contribua à faire de Nakai un théoricien de la communication marxiste. Ueyama Shunpei 上山春平 (1921-2012) souligne quant à lui les liens entre ce même article et les textes théoriques de Mao et en particulier De la pratique (Shijianlun 実践論), tout en faisant remarquer que le travail de Nakai est antérieur (Ueyama 1960 : 58-59). Kuno Osamu ajoute que la réflexion du philosophe, dont il a été très proche dans les années 1930, s'inscrit dans le prolongement d'Histoire et conscience de classe de Lukács (Nakai 1962: 296). Plusieurs auteurs récents, de Monbe Masashi 門部昌志 à Leslie Pincus ont conservé cette image forgée autour de 1960 (Monbe 1998 : 69-74 ; Pincus 2002 : 177), celle d'un intellectuel qui a "assimilé sous une forme originale les principes de Marx et de Lénine ${ }^{17}$ " pour reprendre la formulation du Grand dictionnaire de la littérature moderne japonaise (Nihon kindai bungaku-kan 1984 : 1032).

Il existe cependant d'autres interprétations. Le philosophe Mashita Shin.ichi 真下信一 (1906-1985), qui collabora activement à la revue Sekai bunka, affirme que Nakai n'a jamais été un théoricien matérialiste et le situe à mi-chemin entre positivisme et pragmatisme (Mashita 1975 : 30). La tendance à dissocier Nakai de Marx est particulièrement sensible depuis les années 1990, ce qui n'est pas surprenant. Yoshida Masazumi 吉田 正純 par exemple met l'accent sur d'autres influences. Bien que mentionnant l'environnement marxiste dans lequel baignaient les intellectuels de l'époque, il insiste sur la dette du philosophe à l'égard de Heidegger, notamment en ce qui concerne sa manière de concevoir l'acte et la praxis (Yoshida 2004 : 45-50). Kinoshita Nagahiro 木下長宏, dans une monographie visant à dégager l'originalité propre de Nakai, réduit le marxisme à une référence parmi bien d'autres (Kinoshita 2002 : 91-121). Baba Toshiaki 馬場

16. ほぼあきらかである。

17. マルクス、レーニン主義を独自な形で咀嚼し。 
俊明, dans la biographie très documentée parue en 2009 , tente de montrer qu'il fut avant tout un humaniste bouddhiste qui n'avait aucun lien avec le Komintern (Baba 2009 : 104-195). Harry Harootunian, enfin, voit chez Nakai un penseur néo-kantien et le rattache à un "socialisme non marxien " (non-Marxian socialism; Harootunian 2008 : 99).

Une étude approfondie de « La logique des comités » permet d'apporter de nouveaux éléments de réponse. Cet article, écrit à une époque de répression féroce du communisme, comprend plusieurs citations sans mention d'auteur. Jusqu'à présent celles-ci n’ont jamais été attribuées. On en trouve par exemple une à la fin du passage suivant dont la tonalité marxiste est évidente :

La quantité des moyens de subsistance, qui déterminent la survie de l'individu, doit être suffisante pour renouveler la force de travail. Ceci dit, les besoins naturels de l'homme - des vêtements, de la nourriture, un toit - diffèrent en fonction du climat et d'autres caractéristiques naturelles. L'étendue de ces besoins et les conditions de leur satisfaction sont donc elles-mêmes des données historiques, et déterminent le niveau culturel. Autrement dit, la logique de la technique, qu'on a explicitée comme étant simplement l'ordre humain des fins, en est venue à prendre une forme autoaliénée à force de s'élargir concrètement et de se transformer. La logique de la production est née avec la mise au jour de la rationalité de ce processus. Elle possède la faculté d'auto-aliéner sa propre direction originelle, mais aussi d'évaluer la manière dont s'est réalisée cette possibilité.

Toutefois, nous ne devons absolument pas penser ces divisions sur la base d'un état originel imaginaire (celui d'une sociabilité primordiale par exemple). Car un tel état originel n'explique rien. "Il ne fait que repousser la question dans les brumes confuses et lointaines. [On] suppose comme un fait, comme un événement, ce qu'on doit déduire, à savoir le rapport nécessaire entre deux choses, par exemple entre la division du travail et l'échange. Ainsi le théologien explique l'origine du mal par le péché originel, posant comme un fait, comme un événement historique, ce qu'il doit lui-même expliquer» (Nakai 1981a : 94).

Cette dernière citation est en fait une traduction en japonais d'un passage des Manuscrits de $1844^{18}$. À cette date, ces notes de jeunesse de Marx, qui

18. Le texte original donne : "Er schiebt bloß die Frage in eine graue, nebelhafte Ferne. Er unterstellt in der Form der Tatsache, des Ereignisses, was er deduzieren soll, nämlich das notwendige Verhältnis zwischen zwei Dingen, z.B. zwischen Teilung der Arbeit und Austausch. So erklärt die Theologie den Ursprung des Bösen durch den Sündenfall, d.h., er 
n'ont été rendues publiques pour la première fois qu'entre 1928 et 1932, étaient encore quasiment inconnues au Japon. Elles ne furent traduites qu'en 1964, en même temps que les commentaires de Marcuse ${ }^{19}$. Nakai a donc travaillé directement sur la version allemande. Cette découverte amène de plus à comprendre que le paragraphe qui précède - et en fait tout le chapitre 11 - s'inspire du passage intitulé "Le travail aliéné " (Die entfremdete Arbeit) des Manuscrits de 1844. Les exemples utilisés - "des vêtements, de la nourriture, un toit " - et plus encore le raisonnement suivent étroitement le texte de Marx, ce que personne n’avait imaginé jusqu’à présent, tous les auteurs qui situent Nakai dans une perspective marxiste considérant que ce dernier est arrivé à ces réflexions de façon indirecte, via Lénine, Lukács ou Miki Kiyoshi 三木清 (1897-1945). On comprend désormais que Nakai n’a pas « retrouvé » l'esprit du jeune Marx, il l'a lu et a même pris le risque de le citer afin de laisser une trace de son cheminement intellectuel.

Bien qu'en apparence anodine, cette citation n'a pas été choisie au hasard. Ce que Nakai retient avant tout des Manuscrits de 1844, c'est l'idée qu'il est impossible de donner une origine à la dialectique, qu'on ne peut pas la fonder dans la réalité historique. Car il n'est rien dans le monde qui soit "Un », sauf à retomber dans l'idéalisme hégélien dans lequel Nakai perçoit, malgré l'intérêt qu'il lui témoigne, "l'odeur du sang de la Germanie ${ }^{20}$ " (Nakai 1981a : 62). En refusant, dans une logique radicalement matérialiste, que la logique ait un point de départ, il coupe à la racine tous les mythes fondateurs sur lesquels s'appuyaient à l'époque tant l'impérialisme que le communisme orthodoxe.

Un autre passage se révèle tout aussi passionnant. Situé un peu plus tôt dans l'article, à la fin d'un long développement sur la question du jugement négatif, il donne en français :

Lorsqu'on saisit la manifestation phénoménale d'une essence sous la forme d'un principe ou d'un modèle, ce modèle se retrouve indéfiniment placé face à la question : résiste-t-il ou non à l'examen sur le plan phénoménal d'autres aspects de l'essence ? Ainsi le modèle se rapproche-t-il éternellement de l'essence, tout en étant en

unterstellt als ein Faktum, in der Form der Geschichte, was er erklären soll " (Marx 1932 : 82). Je reprends ci-dessus la traduction de J.-P. Gougeon (Marx 1996 : 108).

19. Voir supra, note 10.

20. ゲルマニヤの血の臭い。 
permanence brisé. "La pensée humaine pénètre sans cesse plus profond du phénomène à l'essence, de l'essence du premier ordre, pour ainsi dire, à l'essence du second ordre, etc., sans fin ». Ce qui importe ici, c'est que cette loi, ce modèle est " un des degrés de la connaissance par l'homme de l'unité et de la liaison, de l'interdépendance et de la totalité du processus universel ». (Nakai 1981a : 76)

Les deux citations, qui sont là encore anonymes, proviennent des Cahiers philosophiques de Lénine. La première - "La pensée humaine... " - est une citation tirée des notes sur les "Leçons d'histoire de la philosophie de Hegel " (1915) (Lénine 1971 : 239). La seconde - « un des degrés de la connaissance..." - est extraite quant à elle des notes sur la "Science de la logique de Hegel » (1914) (Lénine 1971 : 142). Les Cahiers philosophiques du théoricien russe, qui ont été publiés en japonais dès 1932-1933, n'étaient pas inconnus du cercle de Nakai, comme l'indique un article de Miki datant de 1929 (Miki 1967). Ils étaient cependant peu utilisés, non seulement pour des raisons évidentes de sécurité, mais parce qu'ils véhiculent l'image d'un Lénine philosophe et complexe peu conforme à la figure du révolutionnaire prolétarien.

Dans les Cahiers philosophiques, Lénine apparaît moins mécaniciste et dogmatique que dans Matérialisme et Empiriocriticisme (1909) ou Un pas en avant, deux pas en arrière (1904) auquel Tsurumi rattache Nakai. Des passages de Hegel qu'il recopie, et plus encore des notes qu'il prend en marge, se dégage une réflexion sur la négation comme principe fondamental du matérialisme. On n'y trouve pas le caractère téléologique qui domine dans d'autres textes où le processus dialectique tout entier semble dirigé vers le but politique à atteindre (i.e. le monde comme lutte des contraires $\rightarrow$ la société comme lutte des classes $\rightarrow$ la révolution comme suppression des contradictions sociales). Lénine y trace les linéaments d'une description ontologique du monde comme "processus sans fin " où " chaque nouveau stade devient ancien avec le temps ", où " chaque négation est elle-même le théâtre de nouvelles contradictions, le terreau d'une nouvelle négation qui conduit inexorablement vers un nouveau saut qualitatif " (Fryer 1957).

Or tel est précisément, ainsi qu'on l'a vu à travers Tsurumi, l'objectif de départ de Nakai dans "La logique des comités ». Montrer que non seulement l'histoire, mais la logique même est un processus sans origine et sans fin. Que chaque stade de la logique est déjà en soi historique (et inversement), et que chaque négation d'une logique donnée du fait de l'apparition d'un médium nouveau génère un saut vers un stade toujours plus profond 
de développement qui la comprend, mais la transcende. "La logique des comités, écrit Nakai en conclusion de son article, ne pourrait-elle pas se schématiser comme un processus de développement à la fois récursif et infini ?" (Nakai 1981a : 106). Nakai a donc non seulement repéré et compris l'originalité des Manuscrits de 1844, mais il a rapproché les éléments qu'il en a tirés d'un texte de Lénine où ce dernier revient aux origines de la pensée de Marx, à savoir Hegel. Il y a là une cohérence remarquable qui montre bien que Nakai est au milieu des années 1930 un penseur qui s'inscrit dans une ligne assez précise du marxisme, un marxisme hétérodoxe, critique, radicalement immanent, qu'on retrouve, sous des formes diverses, mais convergentes, chez Lukács, Miki, Castoriadis et, bien sûr, chez Horkheimer, Marcuse, Benjamin, Adorno et nombre d'intellectuels qui se rattachent à la théorie critique.

Toutefois l'originalité de Nakai - et notamment par rapport à Miki dont il était proche - est d'avoir compris les implications esthétiques de la dialectique entendue non comme une "méthode " qu'il faut appliquer à un "système " pour le transformer, mais comme le " reflet du développement éternel du monde ", pour reprendre une expression de Lénine (1971 : 108). Bien que "La logique des comités " ne soit pas un texte d'esthétique, sa forme était un enjeu important aux yeux de son auteur. Tout comme le caractère fragmentaire et composite des Manuscrits de 1844 et des Cahiers philosophiques est indissociable du projet critique qui les fondent de remettre la logique hégélienne "sur ses pieds ${ }^{21}$ ", le penseur japonais, qui donne comme sous-titre à son texte "En guise de manuscrit " (Hitotsu no $s \bar{k} k \bar{o}$ to shite 一つの草稿として), prend soin d'emblée de souligner le caractère inachevé, brouillon, bien-vivant de son essai qu'il espère immuniser ainsi contre les méfaits du regard contemplatif. De même, les schémas qu'il propose se présentent davantage comme des moments variants toujours plus élaborés, que comme les tableaux finalisés d'un système philosophique. D'ailleurs, en conclusion, il écrit de façon explicite :

21. Je reprends cette idée à Stathis Kouvélakis dans "Lénine lecteur de Hegel : hypothèses pour une lecture des "Cahiers sur la Science de la Logique de Hegel" ". Texte disponible sur le site du séminaire Marx au XxI ${ }^{\mathrm{e}}$ siècle : http://www.marxau21.fr (dernière consultation : $1^{\text {er }}$ juin 2016). On peut évidemment étendre cette réflexion à Minima Moralia d'Adorno et à l'œuvre de Benjamin en général. 
Ces schémas qui sont proposés ici pour rendre compte de la logique des comités se donnent eux-mêmes comme des propositions, en aucun cas ils ne trouvent leur achèvement sous une forme spéculative; ne pourraient-ils pas trouver une continuité auto-expressive sur le plan du réel si on leur donnait une place à l'intérieur même de la praxis? (Nakai 1981a : 106)

Chez Nakai, esthétique et politique sont par essence indissociables, ce qu'ont très bien perçu les critiques et les artistes des années 1960 qui se sont engagés dans les mouvements de contre-culture précisément pour vivre de façon concrète le rapprochement des mondes de l'art et de l'action sociale.

La cohérence de la position et du projet de Nakai (mais aussi celle de sa réception) apparaît encore plus nettement lorsqu'on découvre les liens qui l'unissent aux philosophes de l'école de Francfort. Sekai bunka, la revue qu'il dirigea entre février 1935 et octobre 1937 avec Kuno, Mashita, Shinmura, mais aussi avec le germaniste Wada Yōichi 和田洋一 (1903-1993) et l'historien Nezu Masashi 襧津正志 (1908-1986), fut l'un des premiers lieux de la réception au Japon des travaux du groupe allemand. Karl Wittfogel servit de relais (Kuno 1986 : 88 ; Siebert 2007 : 40). Wittfogel, historien et économiste marxiste, était le principal spécialiste du monde chinois de l'Institut pour la recherche sociale fondé en 1923 à Francfort. Auteur de nombreux articles dont il tira l'ouvrage qui le rendit célèbre dans les années 1960, Despotisme oriental (1957), il s'installa à Pékin en 1934 pour fuir le régime nazi. Traduit en japonais dès la fin des années 1920 (Kirby 2006 : 57), il fit en 1934 un bref séjour sur l'archipel. Nezu Masashi le rencontra à cette occasion (Nezu 1935 : 9). À la suite de ce premier échange, Nezu écrivit directement à Horkheimer pour lui demander la permission de traduire ses essais en japonais. Horkheimer, comme il le rapporte dans une lettre à Wittfogel du 17 juin 1936, lui donna son autorisation, sous réserve de recevoir le texte avant publication afin de le faire relire par un collègue japonais ${ }^{22}$. Quoique ténue et déséquilibrée, une relation directe a donc existé entre les groupes japonais et allemand.

22. L'échange de courrier entre Nezu et Horkheimer, qu'on peut situer entre janvier et juin 1936, n’a pas été conservé. Il reste en revanche un échange de lettres entre Horkheimer et Wittfogel évoquant la demande de Nezu. Dans une lettre du 17 juin 1936, Horkheimer écrit à son correspondant à Pékin : "Le professeur Nezu 
Masashi du département de littérature de l'université impériale de Kyoto est venu vers moi pour me faire la demande de lui donner la permission de traduire mes essais en japonais. Bien que M. Nezu me soit inconnu, je lui ai accordé mon autorisation de principe, toutefois je lui ai dit que j'aimerais voir une copie du texte avant impression. Je demanderai ensuite l'avis d'un des collègues japonais compétents sur la rigueur de la traduction fournie. Si vous savez vous-même quelque chose sur M. Nezu et pouviez me faire passer un message me disant qui il est, et ce que vous pensez de la question de la traduction, je vous en serais reconnaissant. " (Herr Professor Masashi Nézu von Department of Literature der Kyoto Imperial University hat sich am mich mit der Bitte gewandt, ihm die Erlaubnis zur Uebersetzung meiner Aufsätze ins Japanische zu geben. Obgleich Herr Nézu mir selbst unbekannt ist, habe ich meine Genehmigung grundsätzlich erteilt, jedoch darum gebeten, ein Exemplar des Textes vor der Drucklegung sehen zu dürfen. Ich werde dann einen des Japanischen kundigen Kollegen darum bitten ein Urteil über die Sorgfältigkeit der Uebersetzung abzugeben. Sollten Sie selbst etwa Herrn Nézu kennen und mir eine Mitteilung darüber machen können um wen es sich handelt und was Sie über die Uebersetzungsfrage denken, so wäre ich Ihnen dankbar.)

Wittfogel lui répond le 30 juillet : "En ce qui concerne la question de la traduction, ce que je peux affirmer, c'est que le Dr. N. est un jeune savant ayant reçu une formation universitaire scientifique qui pourra rendre votre travail avec autant de perspicacité qu'on peut en espérer. Je le connais personnellement. Grâce à ses travaux en sociologie et préhistoire, il s'est fait une bonne réputation parmi les jeunes savants les plus en pointe de son pays. Est-il capable de restituer dans sa langue toutes les subtilités de votre raisonnement? Je ne le sais pas, je dirais même que c'est peu probable. Mais vous ne trouverez pratiquement personne capable de faire mieux que le Dr. N. Je pense donc qu'on doit le laisser restituer le canevas de base. Il est déterminé à le faire et le fera avec soin et une implication sincère. Que vous demandiez à voir [les épreuves] avant impression est tout à fait normal. Ceci dit, que cela puisse être effectué en un clin d'œil malgré les milliers de difficultés techniques, seul le Dieu du Temps et de l'Histoire peut en décider." (Was die Frage der Uebersetzung angeht, so kann ich grundsätzlich sagen, dass Dr. N. ein wissenschaftlich hochgeschulter junger Gelehrter ist, der Ihre Arbeit mit so viel Einsicht übertragen wird, wie man nur erwarten kann. Ich kenne ihn persönlich. Er hat sich durch seine soziologischen und prähistorischen Arbeiten einen guten Namen unter den jüngeren und fortgeschritteneren Gelehrten seines Landes gemacht. Ob er alle Feinheiten Ihrer Argumentation in seiner Sprache reproduzieren kann, das weiss ich nicht, ich möchte sogar sagen: es ist unwahrscheinlich. Doch werden Sie kaum jemanden finden, der das im Moment besser vermag als Dr. N. So meine ich, man soll ihn versuchen lassen, das Grundgefüge wiederzugeben. Es wird das bestimmt tun, und er wird es mit Achtung und ehrlicher Bemühung tun. Dass Sie um Einsicht vor der Drucklegung bitten, ist sicher richtig. Ob sich dies freilich im Augenblick wegen der tausend technischen Schwierigkeiten wird durchführen lassen, das kann nur der Gott der Zeit und der Geschichte entscheiden.) Ces lettres sont consultables en ligne sur le site de l'université de Francfort (documents 
Nezu n'avait sans doute pas prévu que Horkheimer exige de recevoir des épreuves et dut en être fort ennuyé. Entre février et mai 1936, Sekai bunka avait en effet publié un long article de Horkheimer, «À propos de la querelle du rationalisme dans la philosophie contemporaine ", dans une traduction de Kuno Osamu (Horkheimer 1936) ${ }^{23}$. On peut en déduire que les rédacteurs japonais n'ont soit pas tenu compte des remarques de Horkheimer, ce qui serait étonnant, soit n'ont pas attendu sa réponse, ce qui paraît plus probable. Outre ce texte, la revue kyotoïte comprend plusieurs articles et comptes rendus sur les activités de l'Institut pour la recherche sociale ${ }^{24}$. Sekai bunka, mensuel diffusé à environ 1000 exemplaires, joua ainsi un rôle pionnier, mais méconnu, dans l'introduction au Japon de Horkheimer, Löwenthal, Marcuse, Benjamin ou encore Mandelbaum (Erich Baumann).

«À propos de la querelle du rationalisme dans la philosophie contemporaine " est considéré comme l'un des plus importants textes du théoricien allemand (Ferry \& Renaut 2009 : 12-18). Horkheimer tente d'y dégager le matérialisme du couple dialectique que constituent depuis Descartes le rationalisme et l'irrationalisme en posant l'amélioration concrète des conditions de vie humaine comme le seul axe à partir duquel la pensée peut valablement s'orienter (Horkheimer $1934: 50 ; 1936$ : [5] 39). Il s'agit d'un texte dense de philosophie sociale qui comprend des attaques directes contre l'individualisme libéral, la glorification du sacrifice individuel et l'organisation du monde en nations. Les valeurs qu'il prône sont à l'opposé de celles qui dominent alors la politique japonaise.

Benjamin a reçu une attention bien moindre que Horkheimer. On ne trouve en effet que deux notules à son sujet, un bref compte-rendu paru

Max Horkheimer, «Korrespondenzen u.a. mit Friedrich Pollock und Karl August Wittfogel », Na 1, 599) :

http://sammlungen.ub.uni-frankfurt.de/horkheimer/content/pageview/6549476

http://sammlungen.ub.uni-frankfurt.de/horkheimer/content/pageview/6549433 (dernière consultation : $1^{\text {er }}$ juin 2016). Je remercie Nicolas Mollard de m'avoir signalé l'existence de ce fonds d'archives numériques.

23. Bien que la traduction de Kuno rende l'essentiel du raisonnement de Horkheimer, quelques phrases et segments sont omis. Il est difficile de savoir si ces omissions sont dues à la difficulté du texte ou à des choix éditoriaux, car elles ne correspondent pas toujours à des passages particulièrement difficiles.

24. Voir en particulier les numéros de novembre 1935 à décembre 1936. 
en novembre 1935 de « La position sociale actuelle de l'écrivain français ${ }^{25}$ " (Kijimoto 1935 : 46-47), et quelques lignes sur " L'œuvre d'art à l'ère de sa reproductibilité technique » en septembre 1936: "L'article de Benjamin, peut-on lire au sujet de ce célèbre essai, traite de la civilisation mécanique depuis 1900 et montre en conclusion que la guerre est son aboutissement. Ce texte critique le manifeste de Marinetti sur la guerre d'Éthiopie et adresse des flèches contre l'exaltation romantique de la guerre ${ }^{26}$ " (Nezu 1936 : 59). Rédigé par Nezu qui n'était guère sensible aux questions esthétiques, ce résumé passe hélas à côté de l'essentiel du texte. Ce sont néanmoins, à ma connaissance, les premières traces d'une lecture de Benjamin au Japon. En dehors de l'école de Francfort, on trouve aussi dans la revue des articles sur (ou traduits de) Heinrich et Thomas Mann, Karl Löwith, Alfred Kantorowicz, Louis Fischer, John Strachey, James Joyce, Romain Rolland, André Gide, Paul Nizan ou encore Antonio Gramsci, autant d'auteurs situés politiquement à gauche, mais souvent en conflit avec le PC (Lucken 2015 : 227-244). On y repère par ailleurs très souvent le nom de Marx, surtout dans les numéros de l'année 1935, et il n'est pas rare d'y rencontrer ceux d'Engels et de Lénine.

Nakai n'a rien écrit sur Horkheimer, ni sur aucun des auteurs liés à l'école de Francfort. Il ne semble pas non plus s'être appuyé sur eux, sauf peut-être dans "Le problème du rationalisme " (Görishugi no mondai 合理 主義の問題, 1937), son dernier essai important avant son arrestation où, non seulement le titre, mais la tonalité générale et certains exemples rappellent le texte de Horkheimer traduit par Kuno quelques mois auparavant (Nakai 1981c). Une étude approfondie étant nécessaire pour étayer cette impression, je la réserve à un prochain article. Quoi qu'il en soit, il est évident qu'il s'intéressait à leurs travaux et sentait un parallélisme avec sa propre pensée. Il partage en effet avec eux, outre des références philosophiques (Kant, Cassirer, Heidegger...), une volonté d'étendre le champ d'application des concepts marxiens de contemplation, naturalisation et

25. L'article de Benjamin fut publié pour la première fois sous le titre "Zum gegenwärtigen gesellschaftlichen Standort des französischen Schriftstellers ", Zeitschrift für Sozialforschung (Revue pour la recherche sociale), 3 (1), Paris, Alcan, 1934 : 54-78.

26. "L'œuvre d'art à l'ère de sa reproductibilité technique " de Walter Benjamin a été publié pour la première fois, en français, dans Zeitschrift für Sozialforschung, 5 (1), Paris, Alcan, 1936 : 40-66. 
fétichisation pour les appliquer de façon critique à la construction même du discours scientifique, ce qui l'a amené à s'intéresser aux rapports entre mesos et topos, milieu et moyen, art et technique, média et communication (Lucken 2015 : 65-93). Il est donc tout à fait logique que nombre d'intellectuels des années 1960-1970, comme on le voit par exemple chez Hariu Ichirō et Tada Michitarō 多田道太郎 (1924-2007), se soient simultanément ou presque intéressés à Nakai et à Benjamin ${ }^{27}$.

\section{La logique du $k i$}

La question du temps est centrale dans l'interprétation que Nakai fait de la logique. Toutefois ce n'est sans doute pas le marxisme qui l'a amené à développer une vision de l'histoire sans origine, sans fin et pourtant toujours susceptible d'être réorientée par l'action immédiate d'individus rassemblés. Des éléments antérieurs, à commencer par son éducation bouddhiste, ont préparé le terrain. Mais il est difficile de résumer l'influence du bouddhisme sur Nakai, car il a grandi à une époque de crise de la pratique religieuse et de transformation des religions existantes en des formes d'expérience intérieure à caractère mystique. Lui-même entra dans un monastère de la Terre pure à l'âge de dix-huit ans, avant de rejoindre au bout d'un an la voie académique. Il en a conservé un rejet de tout ce qui s'apparente à des conceptions magiques. Cependant, il utilise souvent des images et des paraboles bouddhiques. C'est pourquoi on peut considérer que la doctrine de la Terre pure, qui fait de la récitation mantrique le moyen immédiat de l'irruption du sacré, a malgré tout joué un rôle décisif dans sa perception du temps. On peut interpréter de la même manière l'intérêt qu'il porte entre 1928 et 1933 à la phénoménologie de Heidegger.

La réflexion que Nakai a menée tout au long de sa vie autour du concept de $k i$ est un bon moyen de comprendre l'articulation qui existe chez lui

27. Hariu Ichirō est un critique d'art. Il a dirigé la publication des œuvres complètes de Nakai, ainsi que, parallèlement, celle du volume 8 des Écrits de Walter Benyamin (Valutā Benyamin chosakushū ヴァルター・ベンヤミン著作集, vol. 8, Shōbunsha 晶文社, 1981). Tada Michitarō est un philosophe et théoricien des médias. Nakai et Benjamin sont parmi les auteurs qui reviennent le plus régulièrement dans son œuvre (Tada 1994). 
entre action et conscience historique. Le mot japonais $k i$ possède une étymologie complexe. Il se rattache au chinois $q i$, mais il est possible que ce dernier terme se soit agrégé dès le viI ${ }^{\mathrm{e}}$ siècle à un syntagme préexistant en japonais ancien. Dans sa graphie la plus courante 気, il possède un sens très large. Il désigne de façon générale les six éléments primordiaux de la cosmogonie chinoise (le yin, le yang, le vent, la pluie, l'ombre et la lumière) (Nakai 1981b : 202). On le trouve par conséquent dans de nombreux composés désignant des réalités physiques, comme le climat ou la température. Mais il désigne aussi souvent une impression, tantôt concrète, tantôt subjective, qui se dégage des lieux, des êtres et des choses. Il apparaît ainsi dans des composés qui signifient l'ambiance, l'humeur, le charme et même la santé. Sa valeur n'est pas uniquement positive. En particulier lorsqu'on le note avec le caractère 奇, il renvoie à tout ce qui est étrange et mystérieux $^{28}$. Pour résumer, le $k i$ est le mouvement ou souffle interne du monde tel qu'il se donne spontanément, antérieurement à toute rationalisation. Comme le dit Nakai en substance, il désigne la qualité des rapports que les choses entretiennent nécessairement les unes avec les autres (Nakai 1981b : 207-210).

Ce qui est important sur le plan philosophique, c'est que ce concept fonde ontologiquement le sentiment. Le sentiment vu sous cet angle ne relève pas de la contingence, du hasard, voire de la "magie de l'instant ", mais apparaît comme la déclinaison humaine d'une loi essentielle qui veut que tout rapport est nécessairement qualitatif. Il ne saurait par conséquent y avoir de connaissance purement quantitative. Les sciences qui mesurent, numérisent, classent, comparent ne peuvent prétendre à la vérité si elles ne prennent pas en compte le caractère fondamentalement mouvant et qualitatif des rapports qui existent entre les objets dans un champ donné. Ce qui est en jeu pour Nakai est d'abord de faire comprendre qu'il est impossible de dissocier l'objectif du subjectif; la connaissance du sentiment ; l'espace du monde de l'espace de l'être; la technique de l'art. Dans chacun de ces couples, l'un implique nécessairement l'autre. La connaissance scientifique s'articule toujours à un ressenti spécifique. Pour le dire de façon inversée, le ressenti, le rapport qualitatif que l'on établit avec les choses dans toute

28. Nakai mentionne aussi l'expression mononoke (esprit, fantôme) que l'on peut noter soit 物の怪 soit 物の気, ce qui montre une interchangeabilité des caractères 怪 et 気. 
expérimentation n'est pas la part impure de la science qu'il s'agit d'évacuer, mais une donnée de base constitutive d'une vraie connaissance. C'est sans doute Heidegger qui l'amena en premier lieu à cette prise de conscience, mais les développements de Marx sur l'aliénation et la nature - " dès que j'ai un objet, cet objet m'a comme objet... Être matériellement sensible, c'est-à-dire réel, c'est être objet des sens, objet matériellement sensible »-y ont certainement participé aussi (Marx 1996 : 171).

Lorsqu'on considère la situation personnelle de Nakai à la fin des années 1930 et au cours des années 1940, une telle réflexion possède évidemment des implications profondes. Elle présuppose en premier lieu de reconnaître le caractère mouvant du rapport des individus à l'histoire. On ne peut comprendre et analyser des faits historiques qu'à partir de la perception que l'on en a à un moment donné. La peur, l'enthousiasme ou l'indifférence que l'on ressent face à une situation conditionnent l'analyse qu'on en fait. La subjectivité est une loi scientifique. Elle suppose aussi que, contrairement à l'orthodoxie marxiste, la société humaine ne soit pas uniquement orientée par des rapports de production qui évoluent lentement et par palier, mais qu'à chaque moment de crise la polarisation des consciences influe sur le sens des événements. Bien que la réflexion de Nakai sur le ki ait commencé au début des années $1930^{29}$, la poursuite de cette recherche entre 1940 et 1945, à une époque où il a peu écrit, est liée à l'expérience qu'il a faite de la prison et de l'endoctrinement. Parce que l'individu ne peut pas ressentir les choses de la même façon avant et après avoir été violenté, il ne peut pas les comprendre pareillement. Idem à l'échelle collective. Une société a une connaissance de son histoire et de son environnement forcément différente avant et pendant une guerre, parce que son regard sur les choses est modifié par l'évolution des sentiments qu'elle éprouve. Nakai rejette donc non seulement les visions téléologiques qui donnent à l'action une origine et une fin, mais il dit l'impossibilité d'une continuité purement rationnelle, la raison apparaissant toujours prise dans le pathos. On retrouve ici par un autre biais l'idée développée précédemment à propos de " La logique des

29. Le premier article de Nakai sur le concept de $k i$, intitulé "Katagi » 気質 (Tempérament), parut dans le numéro de janvier 1932 (15) de la revue Bi / Hihyó 美・批評 (Beau / Critique) que le philosophe avait lancé en 1930 avec Tsujibe Masatarō 辻部政太郎 (1905-1988) et Nagahiro Toshio 長広敏雄 (1905-1990). 
comités ", à savoir que la logique étant fondamentalement historique, elle implique pour être comprise en elle-même d'être considérée en rapport avec le monde. Étant par essence relative, la vérité d'un moment ne peut jamais être pleinement retrouvée a posteriori, mais elle existe dans l'action présente et se reconnaît à son efficacité sociale.

Cette analyse peut laisser penser que Nakai a cédé au fatalisme qui caractérise une partie des élites intellectuelles japonaises pendant la guerre. Il y a toutefois chez lui une pensée de la liberté qui s'exprime dans sa réflexion sur le $k i$. Dans plusieurs textes, le philosophe explique en effet qu'en japonais classique et notamment à partir de l'époque d'Edo, là où on s'attendrait à rencontrer le caractère habituel $k i$ 気 se trouve parfois un homophone noté 機 $(\mathrm{ch} . \mathrm{ji})^{30}$. Or ce dernier sinogramme signifie un mécanisme ou une opportunité, mais il désigne aussi, dans la terminologie bouddhique, le moi en tant qu'entité propre qui n'a d'autre option que de réagir à la loi universelle. C'est pour Nakai une version asiatique du topos aristotélicien, de l'idée que la logique n'est logique qu'à partir du moment où elle prend appui sur une thèse pour basculer vers une autre. Le fait que ce dernier caractère soit devenu interchangeable avec le premier est l'expression d'une pensée qui est de plus en plus sensible aux moments de crise ( $k e i k i$ 契機, $k i k i$ 危機) et qui, à l'inverse, s'éloigne de l'idée que le monde serait animé par un souffle qui le traverserait de façon continue. En soulignant ce rapprochement, Nakai suggère l'émergence d'une modernité endogène puisque la modernité privilégie par essence les discontinuités, les ruptures, les surgissements. Mais c'est aussi une manière de dire que, si l'individu est soumis à des forces qui le dépassent, il ne peut rester indifférent à ces forces, qu'il réagit nécessairement dans un sens ou dans un autre. Il est par conséquent simultanément l'objet et le sujet de sa réaction, l'objet car il ne peut faire autrement que de réagir et donc de se transformer, et le sujet de celle-ci car à défaut de pouvoir s'y soustraire du moins peut-il l'orienter. C'est dans la capacité de l'homme à orienter à tout instant son rapport au réel que se trouve la liberté chez Nakai. La liberté n'existe pas de façon abstraite, mais dans l'exercice quotidien de ses propres réactions aux événements qui adviennent.

30. Nakai donne en particulier plusieurs exemples tirés du Taiheiki 太平記 (Chronique de la grande paix, XIv siècle) (Nakai $1950: 128)$. 
Cette position n'est pas sans rappeler certains passages de Benjamin. Les convergences entre Nakai et le penseur allemand ont plusieurs fois été soulignées (Takashima 2000 : 52-74 ; Moore 2006 : 244-247). De nombreux points les rapprochent en effet : ils appartiennent à la même génération, ils n'ont pas fait de carrière universitaire, ils furent des pionniers de la réflexion sur la photographie et les nouveaux médias, ils ont attentivement lu Marx, notamment ses écrits de jeunesse, et ils ont été victimes de politiques répressives. La conception de l'histoire de Benjamin est bien connue : l'histoire ne doit pas se résumer à un discours visant à unifier des causes et des effets, non seulement parce que la reconstitution de la chaîne de causalité est forcément erronée, mais parce que cette méthode aboutit toujours à expliquer pourquoi on en est arrivé à telle ou telle configuration sociale. Ce point de vue surplombant qui aplatit les nœuds tend structurellement à aller dans le sens des vainqueurs et des puissants, quand bien même c'est le contraire qui serait dit (Benjamin $2000:$ 431-432). Montrer tel un cheminement logique l'ascension et la ruine de Spartacus, c'est porter en définitive sur lui le même regard que ses bourreaux. C'est pourquoi Benjamin met l'accent sur les moments de tension et tout ce qui relève de la discontinuité. Pour lui, l'historien ne doit pas imaginer le temps comme un continuum, mais au contraire comme une constellation d'arrêts et de blocages (ibid. : 440441). C'est sur ceux-là qu'il doit porter son attention, de sorte à « brosser l'histoire à rebrousse-poil » (ibid. : 433).

Nakai a une formation plus spécifiquement philosophique que Benjamin. Toutefois sa conception du temps témoigne d'un même souci de valoriser le présent comme un moment de tension qui porte en lui la possibilité d'une crise, d'une rupture, d'une réorientation du sens, et non pas comme une simple conséquence du passé, ce qui se ressent dans son écriture où alternent souvent développements structurés et images poétiques. À Benjamin, qui écrit en référence à la tradition hébraïque : "chaque seconde était la porte étroite par laquelle le Messie pouvait entrer» (ibid. : 443), Nakai répond comme en écho sur le thème de "l'unique occurrence sacrée » de l'histoire (sei naru ikkaisei 聖なる一回性 ${ }^{31}$ ), formule dans laquelle

31. Le mot ikkaisei 一回生, néologisme forgé au début du $\mathrm{xx}^{\mathrm{e}}$ siècle pour traduire l'allemand Einmaligkeit, signifie littéralement l'" unique-occurrencité ", c'est-à-dire le " caractère de ce qui n'arrive qu'une fois ". 
on sent l'héritage du bouddhisme de la Terre pure (Nakai 1981d: 171). L'engagement social et politique de Nakai, son souci de toujours traduire sa pensée en acte et de surmonter ses propres défaites sont à comprendre comme une réaction à la propension des hommes à se couler dans une histoire qui n'a pas été écrite pour eux. C'est la raison pour laquelle Tada voit dans son œuvre une "philosophie de l'échappement" (dasshutsu no tetsugaku 脱出の哲学) - l'échappement devant être ici entendu comme le geste de celui qui cherche à s'extraire d'un piège (Tada 1979: 190). On comprend bien comment cette pensée entrait en résonance avec les préoccupations de jeunes intellectuels engagés que le PCJ, en 1959-1960, avait laissés seuls face à la police (Oguma 2009 : 196-217).

Il y a chez Nakai une forme de dialectique négative qui explique la cohérence de sa pensée. Son éducation bouddhique, son expérience de la prison et de la défaite ont ancré en lui l'idée que c'est sur le négatif que se construit la capacité de l'homme à mettre sa pensée en acte. Chez l'homme, dès l'adolescence, la conscience du négatif est tantôt ce qui stimule l'affirmation positive du moi, tantôt ce qui écrase toute velléité d'affirmation individuelle. Or, cette tension génère une réaction qui est aussi un dépassement : " Il arrive que les gens tout à coup se mettent à sourire au moment où ils souffrent le plus ${ }^{32}$ ", écrit Nakai qui voit dans ce sourire de vie au-delà de la défaite l'expression même du ki (Nakai 1948 : 10). Cette réaction est clairement dans son esprit post-traumatique, mais elle n'a rien d'extraordinaire (Kinoshita 2002 : 35). Elle est banale, spontanée et fugace : une "écume blanche " pourrait-on dire en reprenant ce que Bergson écrit dans Le rire (Bergson 1959 : 483). Elle n'est pas le signe de la découverte d'une vérité. Elle n'est qu'un acte ponctuel de lucidité, une réaffirmation transitoire de la force de l'homme, malgré tout. Par essence discontinue, elle n'oriente l'action qu'au moment où elle se manifeste. À l'instar du travail de coupe et de montage dans un film, c'est sa répétition qui fait véritablement sens.

32. 人々は一等苦しいときは、フト微笑することがある。 


\section{Conclusion}

Si Nakai est l'un des précurseurs de la pensée du Japon contemporain, c'est donc que la pensée contemporaine japonaise possède comme caractéristiques d'avoir des origines à la fois kantienne et marxienne, d'être immanente, autoréflexive, engagée, ouverte sur l'avenir, enfin, de concevoir la raison comme une réaction à la négativité du monde, autant d'éléments qui participent des fondements de la théorie critique.

Bien qu'elle ait été souvent et violemment décriée, la théorie critique a répandu son dégrippant dans tous les domaines de la pensée. Partout dans les pays industrialisés, ont été débusqués les mythes des origines, les horizons en carton-pâte, les pseudo-transcendances, les hypostases cachées, et jusqu'à la transformation de la rationalité et de ses outils en fétiches. Elle s'est même retournée contre sa propre famille : l'orthodoxie marxiste, précisément parce qu'elle était devenue orthodoxe, n’y a pas résisté. Bien qu'elle ait commencé à se manifester dès les années 1920-1930, elle s'est surtout imposée à partir des années 1960. En France, on en trouve la trace chez Foucault qui, bien qu'il ait toujours tenu Marx à distance, a compris comment il pouvait s'en servir pour décrire "le surgissement d'un champ social à travers des règles de pure immanence", sans téléologie, "sans qu'il y ait, comme dit Nigro, quelqu'un derrière le rideau " (Nigro 2001 : 444). Baudrillard dans la théorie des médias et de la consommation, Homi Bhabha dans les études post-coloniales, Judith Butler dans les études de genre ont contribué à poursuivre ce travail au sein de leur domaine respectif.

Plusieurs des grands intellectuels japonais des années 1960 s'inscrivent dans ce courant. Ils ont relu Kant, Hegel et Marx contre le communisme et les pensées mythologiques, et se sont volontiers appuyés sur les différentes traditions bouddhiques pour développer une vision radicalement immanente de l'histoire. Les mouvements protestataires en sont l'expression socio-politique. Ils transformèrent la société de l'intérieur à travers d'innombrables mouvements de libération - politique à Okinawa, sociale à Miike, sexuelle et artistique à Osaka, Tokyo et Fukuoka, environnementale à Narita, etc. -, mais ne purent se mettre d'accord sur une alternative globale. Ils ont ouvert une ère où ce qui compte n'est plus de faire aujourd'hui pour être demain, comme l'ont proposé toutes les pensées téléologiques, religieuses ou profanes, mais de faire de sorte que, du mouvement même, puisse apparaître une autre conception de l'être, rhizomique et instable, 
libre et connectée. L'esthétique relationnelle qui s'est développée avec force au Japon dans les années 1970 à travers un groupe comme Mono-ha もの派 par exemple en est l'un des développements saillants.

Toutefois le paradoxe est le suivant. Alors que la théorie critique s'est construite dans le rejet de toutes les idéologies qui hypostasiaient une race, un territoire, des origines, mais aussi de la Science, de l'abstraction analytique et de toutes les formes de domination «logique", renvoyant dos à dos l'irrationalisme et le rationalisme, depuis les années 1960-1970 et la prise de conscience de l'échec des tentatives staliniennes et maoïstes, et plus encore depuis 1990 et la dissolution de l'empire soviétique, elle a perdu de son efficacité car le système économique aujourd'hui dominant n'a plus besoin de convoquer de grandes figures de vérité pour asseoir sa légitimité. La fluidité du capitalisme financier, sa nature puissamment opportuniste et mensongère le rendent quasiment insensible à la déconstruction critique.

La contemporanéité de Nakai réside en partie dans ce paradoxe. Nakai est contemporain parce que son œuvre permet de s'attaquer aux camps retranchés, aux fétiches, aux sanctuaires comme il l'a remarquablement fait lui-même avec la Loi sur les bibliothèques de 1950, mais il est contemporain aussi parce que la théorie critique dont il a été l'un des précurseurs non seulement est soluble dans la forme actuelle du capitalisme sur laquelle elle n'a pas de prise, mais fait porter la responsabilité de rebondir et de donner du sens sur les individus vaincus. Ce n'est peut-être pas un hasard si, à l'heure où la spéculation bat son plein dans le marché de l'art contemporain, les œuvres de Benjamin dominent le monde de la critique. 


\section{Bibliographie}

\section{ANDO Takemasa 2013}

Japan's New Left Movements. Legacies for Civil Society, Oxon, Routledge.

\section{BABA Toshiaki 馬場俊明 2009}

Nakai Masakazu densetsu : nijūichi no shōzō ni yoru yūwaku 中井正一伝説 :

二十一の肖像による誘惑 (La légende de Nakai Masakazu : une tentation en vingt et un portraits), Tokyo, Potto shuppan ポット出版.

\section{BENJAMIN Walter 2000 [1991]}

«Sur le concept d'histoire », Fuvres, 3, Paris, Gallimard : 427-443.

\section{BERGSON Henri 1959}

« Le rire », Fuvres, Paris, PUF : 383-483.

\section{CAPEL Mathieu 2012}

Dans les coupures du monde. Cinéastes japonais face à la haute croissance 19561973, thèse de doctorat, université Paris 3.

\section{CAPEL Mathieu 2015}

Évasion du Japon. Cinéma japonais des années 1960, Paris, Les prairies ordinaires.

\section{FERRY Luc \& RENAUT Alain 2009}

"Présentation », in Horkheimer Max, Théorie critique, Paris, Payot : 7-32.

\section{FRYER Peter 1957}

«Lenin as Philosopher », Labour Review, 9/10.

http://www.marxistsfr.org/archive/

fryer/1957/09/lenin-phil.html
HAROOTUNIAN Harry D. 2008

"Time, Everydayness and the Specter of Fascism: Tosaka Jun and the Philosophy's New Vocation ", in Christopher Goto-Jones (ed.), Re-Politicising the Kyoto School as Philosophy, Oxon, Routledge : 96-112.

\section{HORKHEIMER Max 1934}

« Zum Rationalismusstreit in der gegenwärtigen Philosophie », Zeitschrift für Sozialforschung, 3 (1), Paris, Alcan : 1-53.

\section{HORKHEIMER Max 1936}

«Gendai tetsugaku ni okeru gōrishugi no ronsō » 現代哲学における合理主義 の論争 (À propos de la querelle du rationalisme dans la philosophie contemporaine), trad. Kuno Osamu 久野収 (alias Matsuo Shirō 松尾史郎), Sekaibunka 世界文化, 2 : 34-41; 3 : 26$33 ; 4: 21-30 ; 5: 31-41$.

\section{HOWARD Dick 1970}

« Fétichisme, aliénation et théorie critique : réflexion sur un manuscrit de Marx publié récemment ", L'homme et la société, 17 (7) : 97-110.

KARATANI Kōjin (dir.) 柄谷行人 1997 Kindai Nihon no hihyō 近代日本の批評 (La critique du Japon moderne), 1, Tokyo, Kōdansha 講談社.

\section{KAWAZOE Noboru 川添登 1965}

Nihon bunka to kenchiku 日本文化と建築 (La culture japonaise et l'architecture), Tokyo, Shōkokusha 彰国社. 
KAWAZOE Noboru 1981

«Shisō sensen no senkun toshite » 思想 戦線の戦訓として (Les leçons du Front des idées), Nakai Masakazu zenshū, 3, livret annexe, Tokyo, Bijutsu shuppansha 美術 出版社 : 12-15.

\section{KIJIMOTO Hiroshi 雉本浩 1935}

« Kaigai bunka shiryō : Shakai kenkyū zasshi ni tsuite » 海外文化資料「社会研究 雑誌」について (Documents sur la culture à l'étranger : La revue pour la recherche sociale), Sekaibunka 世界文化, 11, Kyoto, novembre 1935 : 46-47.

\section{KINOSHITA Nagahiro 木下長宏 2002}

Nakai Shōichi : atarashii "bigaku" no kokoromi-zōho 中井正一 : 新しい「美学」 の試み 増補 (Nakai Shōichi : la tentative d'une nouvelle « esthétique » - version augmentée), Tokyo, Heibonsha 平凡社.

\section{KIRBY E. Stuart 2006 [1954]}

An Introduction to the Economic History of China, London, Routledge.

KŌYAMA Iwao 高山岩男 1995

Kyōto tetsugaku no kaisō. Kyūshi kyūyū no tsuioku to waga shisaku no kiseki

京都哲学の回想 : 旧師旧友の追憶とわが

思索の軌跡 (Retour sur la philosophie de Kyoto. Souvenirs de mes maîtres et amis, et linéaments de ma réflexion), Kyoto, Tōeisha 燈影舎.

\section{KUNO Osamu 久野収 1986}

Fashizumu no naka no 1930 nendai

ファシズムの中の一九三○年代 (Les années 1930 en plein fascisme), Tokyo, Riburopōto リブロポート.
KUWABARA Takeo 桑原武夫 1962

Nihon no meicho. Kindai no shisō 日本 の名著 : 近代の思想 (Les grands textes du Japon. La pensée moderne), Tokyo, Chūō kōron sha 中央公論社.

Kyōdō kumiai-shi kenkyūkai 協同組合史 研究会 (éd.) 2003

Nose Katsuo to Kyōto (katei) shōhi kumiai 能勢克男と京都(家庭)消費組合 (Nose Katsuo et la Coopérative familiale de Kyoto), Kyoto, Kurashi to kyōdō no kenkyūjo くらしと共同の研究所.

\section{LÉNINE Vladimir Ilitch 1971}

Cahiers philosophiques, Fuvres, 38, Paris, Éditions sociales.

\section{LUCKEN Michael 2014}

«Les limites du ma: retour à l'émergence d'un concept "japonais" », Nouvelle revue d'esthétique, 13 (1) : 45-67.

\section{LUCKEN Michael 2015}

Nakai Masakazu. Naissance de la théorie critique au Japon, Dijon, Presses du réel.

\section{LUCKEN Michael 2016}

Les Fleurs artificielles. Création, imitation et logique de domination, Paris, Presses de I'Inalco.

\section{MARX Karl \& ENGELS Friedrich 1932}

Historisch-kritische Gesamtausgabe: Werke, Schriften, Briefe, Abt. 1, Bd. 3, Berlin, Marx-Engels-Lenin-Institut.

\section{MARX Karl 1996}

Manuscrits de 1844, trad. J.-P. Gougeon, Paris, Flammarion. 
MASHITA Shin.ichi 真下真一 et al. 1975 «Zadankai : Sekai bunka no koto» 座談会『世界文化』のこと (Table ronde : Culture du monde), Sekai bunka : fukkoku 世界文化 復刻 (Culture du monde : fac-similé), Tokyo, Shōgakukan 小学館 : 15-47.

\section{MATSUMOTO Toshio 松本俊夫 2006} [1967]

« Nakai Masakazu no eigaron » 中井正一 の映画論 (La théorie du cinéma de Nakai Masakazu), Hyōgen no sekai. Geijutsu zen.ei tachi to sono shisō 表現の世界— 芸術前衛たちとその思想 (Le monde de l'expression. Les avant-gardes et leur pensée), Tokyo, Seiryū shuppan 清流 出版 : 238-239.

\section{MIKI Kiyoshi 三木清 1967}

« Hēgeru no benshōhō to Rēnin » ヘーゲルの弁証法とレーニン (La dialectique de Hegel et Lénine), Miki Kiyoshi zenshū (Fuvres complètes de Miki Kiyoshi), 10, Tokyo, Iwanami shoten 岩波書店 : 77-82.

MONBE Masashi 門部昌志 1998 « Nakai Masakazu kenkyū to media shakaigaku no shiten » 中井正一 研究とメディア社会学の視点 (Les études sur Nakai Masakazu et le point de vue de la sociologie des médias), Shakai kankei kenkyū 社会関係研究, 4 (2), Kumamoto gakuen daigaku 熊本学園 大学: 69-95.

\section{MOORE Aaron 2006}

The Technological Imaginary of Imperial Japan, 1931-1945, PhD dissertation, Cornell University.
NAKAI Masakazu 中井正- 1948 "Gendai nihonga no hitotsu no kadai » 現代日本画の一つの課題 (Une question qui se pose à la peinture contemporaine de style japonais), Sansai 三彩, 1, Tokyo, Nihon bijutsu shuppan 日本美術出版: 8-12.

\section{NAKAI Masakazu 1950}

«Gengo wa ikite iru » 言語は生きてるる (Les mots sont vivants), Chūō kōron 中央 公論, 742 (12) : 126-130.

\section{NAKAI Masakazu 1951}

Bigaku nyūmon 美学入門 (Introduction à l'esthétique), Tokyo, Kawade shobō 河出書房.

\section{NAKAI Masakazu 1962}

Bi to shūdan no ronri 美と集団の論理 (Logique du beau et logique collective), Tokyo, Chūō kōronsha.

\section{NAKAI Masakazu 1981a}

《 linkai no ronri » 委員会の論理 (La logique des comités), Nakai Masakazu zenshū 中井正一全集, 1, Tokyo, Bijutsu shuppan 美術出版 : 46-108.

\section{NAKAI Masakazu 1981b}

《Katagi » 気質 (Tempérament), Nakai Masakazu zenshū 中井正一全集, 2, Tokyo, Bijutsu shuppan 美術出版 : 199-210.

\section{NAKAI Masakazu 1981c}

《Gōrishugi no mondai » 合理主義の問題 (Le problème du rationalisme), Nakai Masakazu zenshū 中井正一全集, 1，Tokyo， Bijutsu shuppan 美術出版 : 123-142. 
NAKAI Masakazu 1981d

«Kaiga no fuan » 絵画の不安 (L'angoisse de la peinture), Nakai Masakazu zenshū 中井正一全集, 2, Tokyo, Bijutsu shuppan 美術出版 : 169-178.

\section{NEZU Masashi 襧津正志 1935}

«Wittofōgeru hakase no Nihon hōmon » ウィットフォーゲル博士の日本訪問 (Visite au Japon du Docteur Wittfogel), Shinagaku 支那學, 8 (1): 9.

\section{NEZU Masashi 1936}

«Shinkan hihyō » 新刊批評 (Critique des nouvelles parutions), Sekai bunka 世界 文化, 21, septembre 1936: 59

\section{NIGRO Roberto 2001}

«Foucault lecteur et critique de Marx ", in Jacques Bidet \& Eustache Kouvélakis (dir.), Dictionnaire Marx Contemporain, Paris, PUF : 433-446.

\section{Nihon kindai bungaku-kan 日本近代} 文学館 (ed.) 1984

Nihon kindai bungaku daijiten 日本近代 文学大事典 (Grand dictionnaire de la littérature moderne japonaise), Tokyo, Kōdansha 講談社.

OGUMA Eiji 小熊英二 2009 1968 - Wakamonotachi no hanran to sono haikei 1968 若者たちの叛乱とその 背景 (1968 - La révolte des jeunes et son contexte), 1, Tokyo, Shin.yōsha 新曜社.

\section{PINCUS Leslie 2002}

«A Salon for the Soul: Nakai Masakazu and the Hiroshima Culture Movement ", Positions: Asia critique, 10 (1), Duke University Press : 173-194.

\section{PINCUS Leslie 2006}

« Revolution in the archives of memory: the founding of the National Diet Library in Occupied Japan », in Francis X. Blouin \& William G. Rosenberg (eds.), Archives, Documentation and Institutions of Social Memory: Essays from the Sawyer Seminar, Ann Arbor, University of Michigan Press : 382-392.

\section{SATŌ Shin.ichi 佐藤晋一 1992}

Nakai Masakazu : "toshokan" no ronrigaku 中井正一・図書館」の論理学 (Nakai Masakazu : la logique des bibliothèques), Tokyo, Kindai bungeisha 近代文芸社.

\section{SATŌ Yuichi 2012}

Kyoto Popular Front. Anti-fascist movement at a café in Kyoto during the 1930s, Kyoto, Hokuto shobo books.

\section{SIEBERT Rudolf 2007}

«Introduction », in Michael R. Ott (ed.), The Future of Religion: Toward a Reconciled Society, Leiden, Brill : 1-70.

TADA Michitarō 多田道太郎 1979 Teihon : Kanri shakai no kage 定本 管理社会の影 (L'ombre de la société sous contrôle : le livre de base), Tokyo, Nihon buritanika 日本ブリタニカ.

\section{TADA Michitarō 1994}

Tada Michitarō chosakushū 多田道太郎 著作集 (Écrits de Tada Michitarō), 6 vol., Tokyo, Chikuma shobō 筑摩書房.

TAKASHIMA Naoyuki 高島直之 2000 Nakai Masakazu to sono jidai 中井正一と 
その時代 (Nakai Masakazu et son temps), Tokyo, Seikyūsha 青弓社.

TSURUMI Shunsuke 鶴見俊輔 1959 «Shisō no hakkō botai » 思想の発酵 母胎 (La matrice de fermentation de la pensée), Shisō no kagaku 思想の科学, 7, Tokyo : 30-39.

TSURUMI Shunsuke et al. 1963

«Zadankai : Nakai Masakazu to wareware no jidai » 座談会 中井正一と われわれの時代 (Nakai Masakazu et notre époque), Shisō no kagaku 思想の科学, 14 (5), Tokyo : 71-89.

\section{TSURUMI Shunsuke 2009}

"Taido to chishiki : Shisō no kagaku shōshi » 態度と知識『思想の科学』小史 (Comportement et savoir : brève histoire de Shisō no kagaku), Shisō 思想, 5, Tokyo, Iwanami shoten 岩波書店 : 2-6.

\section{UEYAMA Shunpei 上山春平 1960}

"Nakai Masakazu no "linkai no ronri" » 中井正一の「委員会の論理」(Table ronde : «La Logique des comités » de Nakai Masakazu), Shisō no kagaku 思想の科学， $23: 55-60$.

YOSHIDA Kijū 吉田喜重 2006

« Eiga ni okeru kūkan to jikan » 映画に おける空間と時間 (L'espace et le temps au cinéma), Henbō no rinri 変貌の倫理 (Éthique de la métamorphose), Tokyo, Seidosha 青土社 : 257-280.

YOSHIDA Masazumi 吉田正純 2003 "Seikatsu ni taisuru yūki : zenhen » 生活に対する勇気 前編 (Le courage face à la vie : première partie), Kyōto daigaku shōgai kyōiku-gaku toshokan jōhō-gaku

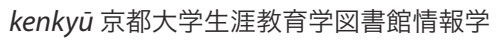
研究, 2, Kyōto daigaku : 7-38.

\section{YOSHIDA Masazumi 2004}

"Seishin no meiseki : "Sekai bunka" shūdan no teikō to gakushū » 精神の明晰 『世界文化』集団の抵抗と学習 (La lucidité de l'esprit : résistance et apprentissage du groupe Culture du monde), Kyōto daigaku shōgai kyōiku-gaku toshokan jōhō-gaku kenkyū 京都大学生涯教育学 図書館情報学研究, 3, Kyōto daigaku : 35-59.

\section{YOSHIKAWA Mitsusada 1952}

«From Mitsusada Yoshikawa / For GS, GHQ / On Nakai Shoichi », GHQ/SCAP Records, Government Section (GS), GS(B)03380, 30/01/1952.

YOSHIMOTO Takaaki 吉本隆明 1966 Kāru Marukusu カール・マルクス (Karl Marx), Kōriyama, Shikō shuppanbu 試行出版部. 